\title{
Dynamical Analysis and Circuit Design for Malasoma System
}

\author{
Volodymyr Rusyn ${ }^{1, *}$, Diana Purwandari ${ }^{2}$ \\ ${ }^{1}$ Department of Radio Engineering and Information Security, \\ Yuriy Fedkovych Chernivtsi National University, Chernivtsi, Ukraine \\ ${ }^{2}$ Department of Mining Engineering, Universitas Muhammadiyah Tasikmalaya, Indonesia \\ *Corresponding author email: rusyn_v@ukr.net
}

\begin{abstract}
In this paper, the Malasoma system based cubic function is presented. This system contains operational amplifiers, resistors, capacitors, multipliers, and voltage sources. The first stage, we analyze the Malasoma model and execute its stability. The phase portraits and bifurcation diagram are used to analyze the dynamic behaviors of the Malasoma model. The proposed circuit was modelled by utilizing NI's MultiSim software environment. The electronic circuit is realized by using off-the-shelf components. MATLAB and MultiSim simulation results show a good agreement.
\end{abstract}

Keywords: chaos, dynamical system, Malasoma system, circuit design

\section{Introduction}

Chaos is a study that is very intensively researched by scientists in various disciplines such as robotic (Vaidyanathan et al., 2017), video encryption (Preishuber et al., 2020), image encryption (Sambas et al., 2020), sound encryption (Mobayen et al., 2019), touchless fingerprint encryption (Lai et al., 2020), network encryption card (Ding et al., 2007), Permanent magnet synchronous motor (Yin et al., 2020), homopolar dynamo system (Chen et al., 2020), Rotor-disk-Bearing system (Haghani and Safarpour, 2018), electronic circuit (Sambas et al., 2019), neuron network system (Zhou et al., 2008), tumor-immune model (Kumar et al., 2020), epidemiological model (Ghezzi and Piccardi, 1997), financial risk (Sukono et al., 2020) and regular longitudinal waves (Hu et al., 2010).

Sambas et al. (2015) studied behavior of Malasoma system and its application in voice encryption. They show that the Malasoma system is very well used in voice encryption with AWGN techniques. Varan et al. (2016) presented Malasoma system with P-C synchronization and 
its application in secure communication system. They have successfully synchronized and implemented a communication security system on Malasoma model using MATLAB-Simulink. However, the researchers found it very difficult to apply the Malasoma model to electronic circuits. Therefore, we will solve this problem with Kirchhoff's legal assistance.

In this study, the Malasoma model is introduced and its dynamical properties are investigated. In Sec. 2, the existence of chaotic behavior in this system is demonstrated. In Section 3, In Section 3, we determine complex behavior, and introduce the MultiSim platform, together with the electronic circuit schema of the system.

\section{Mathematical Model and Dynamical Analysis}

Malasoma chaotic system, which was proposed by Malasoma (2000) is described by the following chaotic system with only one cubic nonlinear term

$$
\begin{aligned}
& \dot{x}=y \\
& \dot{y}=z \\
& \dot{z}=-a z+x y^{2}-x
\end{aligned}
$$

Where $x, y, z$ are a variable and when $a=2.05$, the Malasoma system in equation (1) shows strange attractors, we have chosen initial conditions for the Malasoma system $\left(x_{0}, y_{0}, z_{0}\right)=(0,0.96$, $0.1)$. The Lyapunov exponents are $(0.0541,0,-2.1041)$, and the Kaplan-Yorke dimension is $D_{\mathrm{KY}}=$ 2.0257 (Sprott and Linz, 2000).

For numerical simulations of a Malasoma system defined by a set of differential equations, such as the Malasoma system in equation (1), different integration techniques can be used. In Figures 1 (a) - (c), respectively, show the projections of the orbital space of the $x$-y plane phase, the $x-z$ plane and the $y-z$ plane. For the selected parameter set and initial conditions, the Malasoma system in equation (1) present a dense strange attractor. By using bifurcation diagram (See Figure 2), the range of a over which chaos occurs is very narrow, $2.02<a<2.08$. Also, small window of chaos for $0.075351<a<0.075362$.

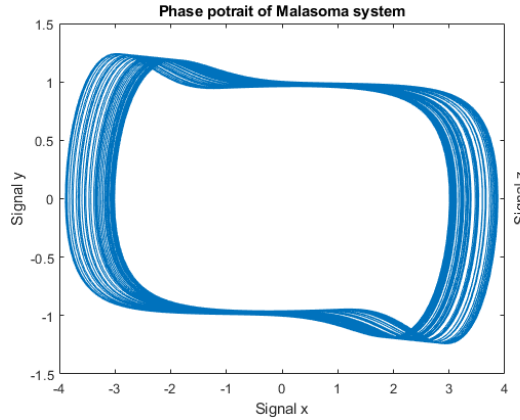

(a)

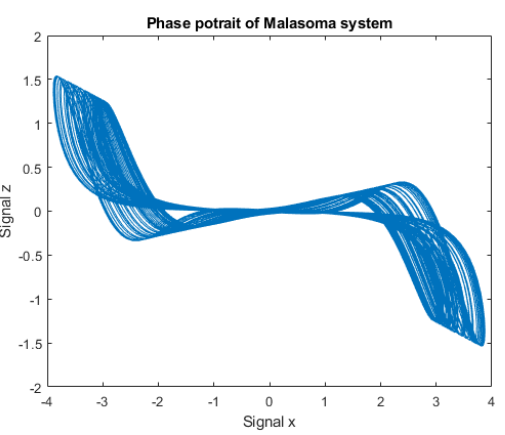

(b)

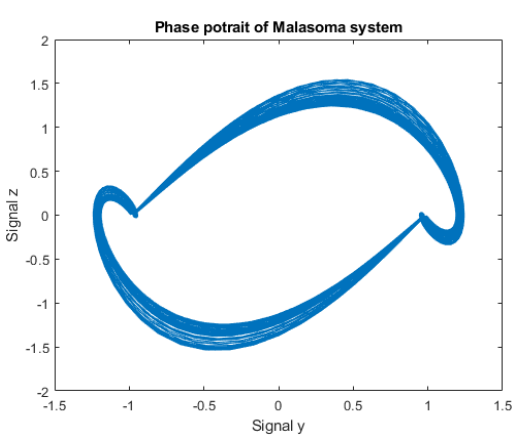

(c)

Figure 1. Numerical simulation results using MATLAB, with $a=2.05$ in the (a) $x$-y plane, (b) $x-z$ plane, and (c) $y-z$ plane 


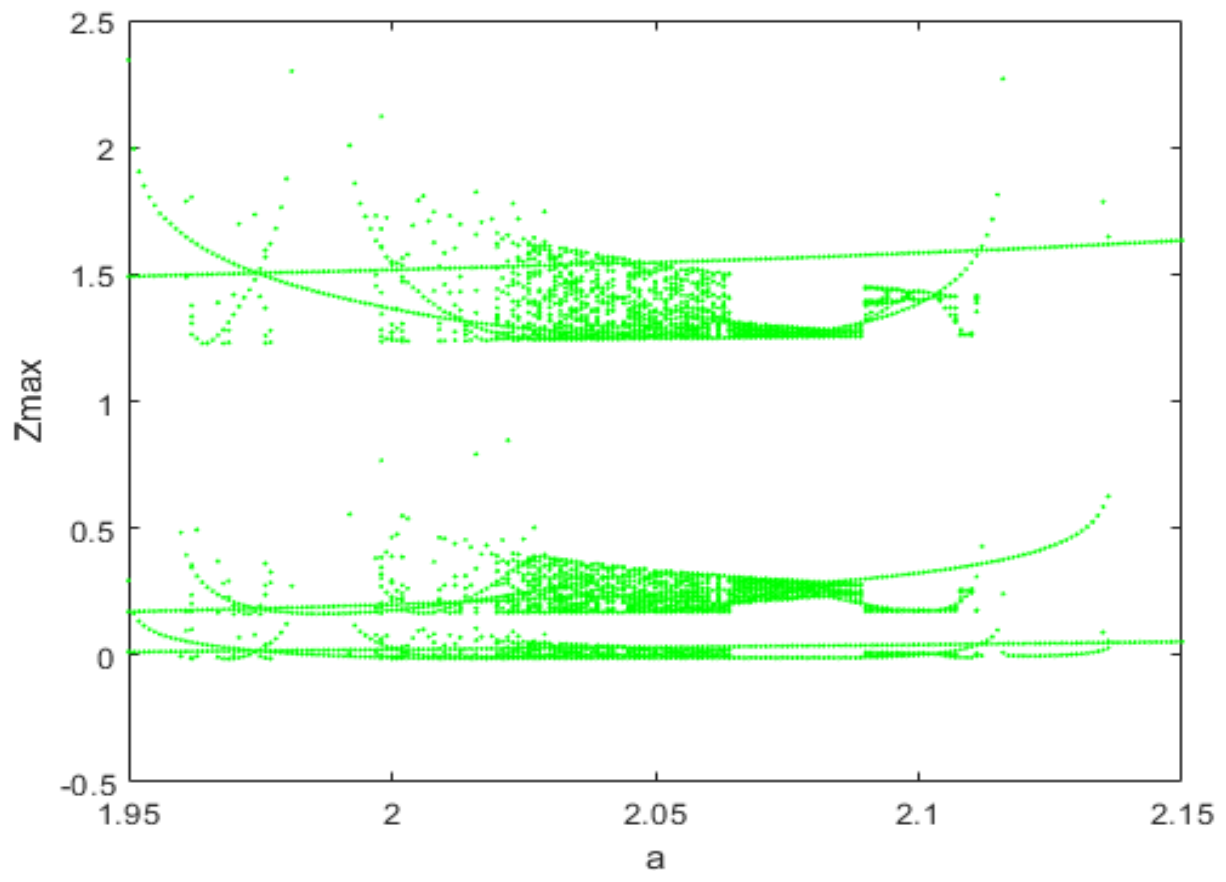

Figure 2. Bifurcation Diagram (between 1.95-2.15).

The equilibria of the Malasoma system (1) are found by setting $\dot{x}=\dot{y}=\dot{z}=0$, i. e.,

$$
\begin{aligned}
& 0=y \\
& 0=z \\
& 0=-a z+x y^{2}-x
\end{aligned}
$$

The Malasoma system has one equilibrium point $E_{0}(0,0,0)$. For the equilibrium point $E_{0}(0,0$, 0 ), the Jacobi matrix becomes:

$$
J(0,0,0)=\left[\begin{array}{ccc}
0 & 1 & 0 \\
0 & 0 & 1 \\
-1 & 0 & -2.05
\end{array}\right]
$$

To obtain its eigenvalues, let det $\left|J_{1}-\lambda\right|=0$. Then, the characteristic equation has the following form:

$$
\lambda^{3}+2.05 \lambda^{2}+1
$$


Solving the above characteristic equation, the eigenvalues are found as $\lambda_{1}=-2.2479, \lambda_{2,3}=-0.0989 \pm 0.6595$

Here $\lambda_{1}$ is a negative real number, $\lambda_{2}$ and $\lambda_{1}$ are conjugate pair of complex eigenvalues having positive real parts. That means the equilibrium $E_{0}(0,0,0)$ is a saddle point. So, this equilibrium point is unstable

\section{Electronic Circuit}

The analog circuit of the Malasoma system was designed in MultiSim. The analog circuit of the Malasoma system is seen in Figure 2. The circuit includes simple electronic elements such as resistors, operational amplifiers.

For circuit implementation, we rescale the state variables of the Malasoma system (1) as follows: $X=6 x, Y=2 y, Z=2 z$. The rescaled chaotic system is given as follows:

$$
\begin{aligned}
& \dot{X}=3 Y \\
& \dot{Y}=Z \\
& \dot{Z}=-a Z+\frac{X Y^{2}}{12}-\frac{X}{3}
\end{aligned}
$$

Applying the Kirchhoff laws, the circuit presented in Figure 3 is described by the following equations:

$$
\begin{aligned}
& \dot{X}=\frac{1}{C_{1} R_{1}} Y \\
& \dot{Y}=\frac{1}{C_{2} R_{2}} Z \\
& \dot{Z}=-\frac{1}{C_{3} R_{3}} Z+\frac{1}{100 C_{3} R_{4}} X Y^{2}-\frac{1}{C_{3} R_{5}} X
\end{aligned}
$$

We get the value of electronic components $R_{1}=200 \mathrm{k} \Omega, R_{2}=600 \mathrm{k} \Omega, R_{3}=292.68 \mathrm{k} \Omega, R_{4}=72$ $\mathrm{k} \Omega, R_{5}=1.8 \mathrm{M} \Omega, R_{6}=R_{7}=R_{8}=R_{9}=R_{10}=R_{11}=100 \mathrm{k} \Omega$, and $C_{1}=C_{2}=C_{3}=1 \mathrm{nF}$. Figure 4 illustrates phase portraits which are obtained from the designed circuit. As it can be seen from the MultiSim outputs in Figure 4 and MATLAB simulation in Figure 1, the results are similar. 

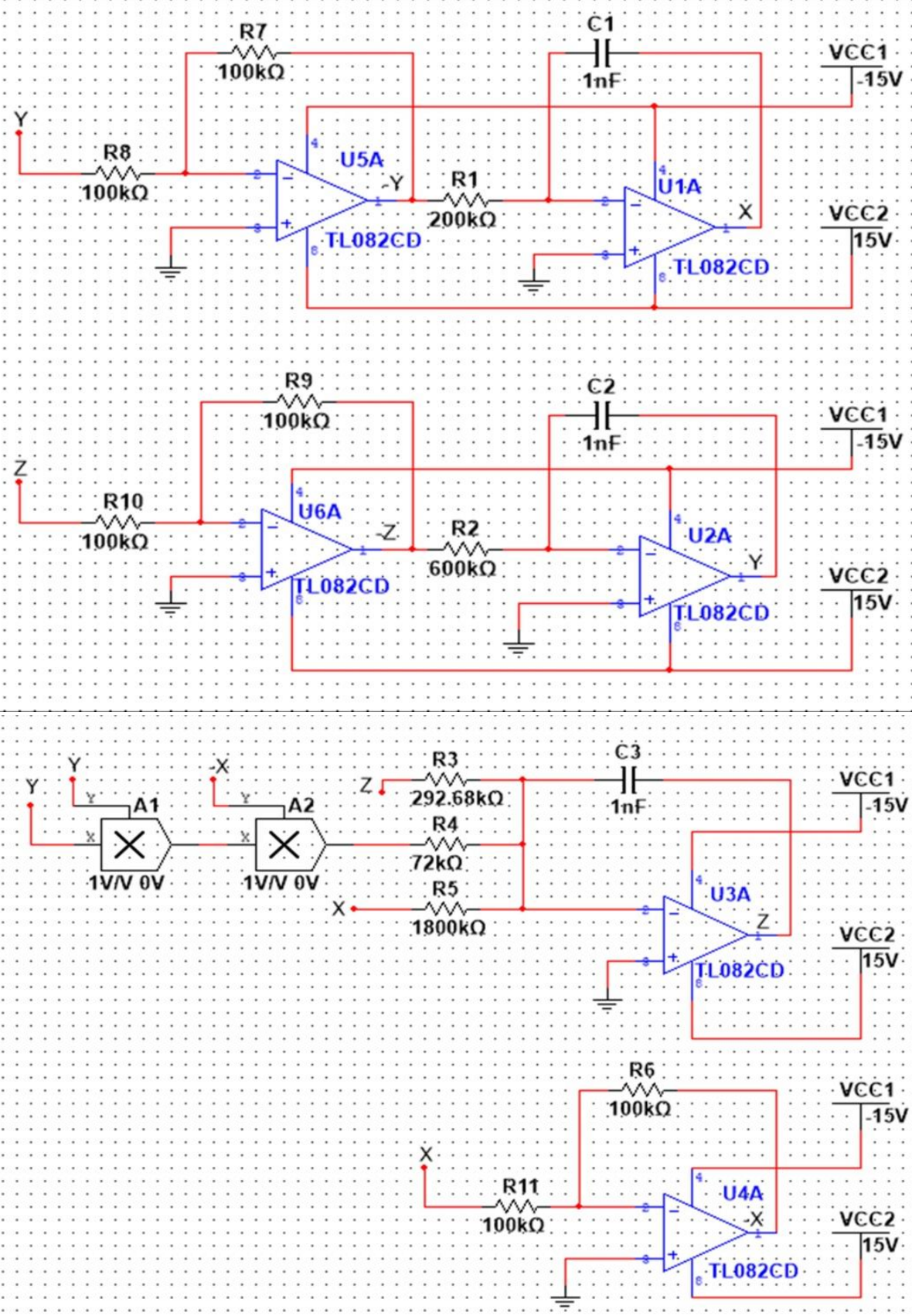

Figure 3. The electronic circuit schematic of the Malasoma system 


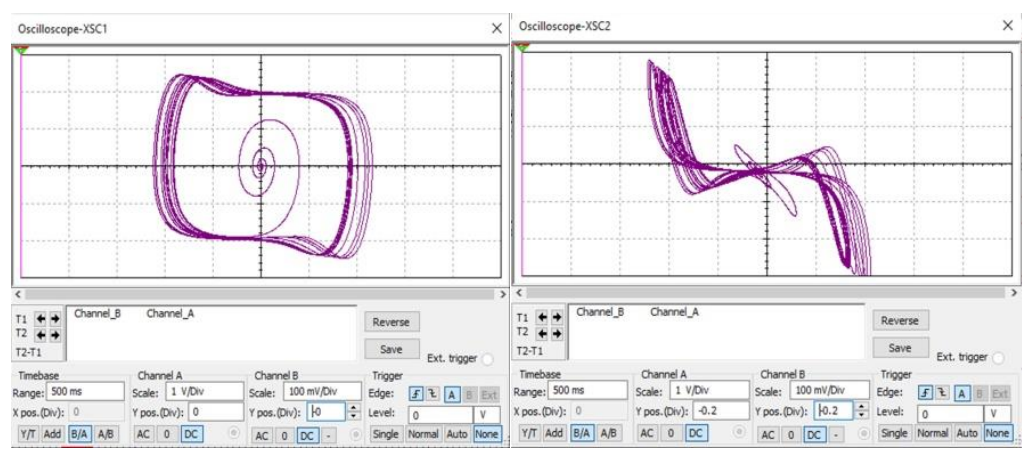

(a)

(b)

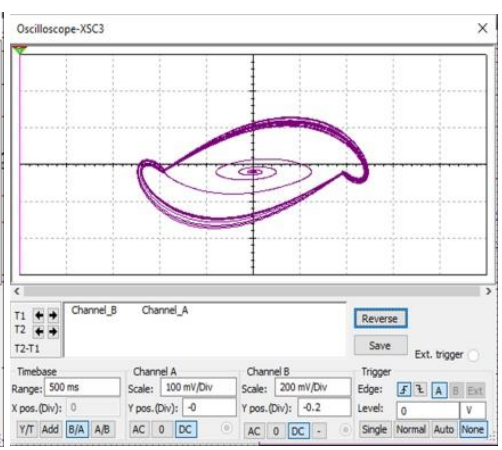

(c)

Figure 4. Multisim outputs of the in the Malasoma system (a) $X-Y$ plane, (b) $X-Z$ plane, and (c) $Y-Z$ plane

\section{Conclusion}

In this study, we have studied a Malasoma model with cubic function which is obtained obtained by Malasoma (2000). Fundamental dynamical properties of the Malasoma model have been discovered through equilibrium point analysis, bifurcation diagram and Lyapunov exponents. Finally, the MATLAB simulations and MultiSim simulation are performed to verify the theoretical model. For hardware electronic circuit can be investigated in our future works.

\section{References}

Ghezzi, L. L., and Piccardi, C. (1997). PID control of a chaotic system: An application to an epidemiological model. Automatica, 33(2), 181-191.

Haghani, A., and Safarpour, P. (2018). The Effect of System Parameters on the Chaotic Behavior of Rotordisk-Bearing with Rubbed between the Disk and the Stator, University of Tabriz Journal of Mechanical Engineering, 81(18), 125-133.

Hu, K. Y., Ding, Y., and Wang, H. W. (2010). Chaotic roll motions of ships in regular longitudinal waves. Journal of Marine Science and Application, 9(2), 208-212.

Kumar, S., Kumar, A., Samet, B., Gómez-Aguilar, J. F., and Osman, M. S. (2020). A chaos study of tumor and effector cells in fractional tumor-immune model for cancer treatment. Chaos, Solitons \& Fractals, 141, 110321.

Lai, Q., Wan, Z., Akgul, A., Boyraz, O. F., and Yildiz, M. Z. (2020). Design and implementation of a new memristive chaotic system with application in touchless fingerprint encryption. Chinese Journal of Physics, 67, 615-630.

Malasoma, J. M. (2000). What is the simplest dissipative chaotic jerk equation which is parity invariant?. Physics Letters A, 264(5), 383-389. 
Mobayen, S., Vaidyanathan, S., Sambas, A., Kacar, S., and Çavuşoğlu, Ü. (2019). A novel chaotic system with boomerang-shaped equilibrium, its circuit implementation and application to sound encryption. Iranian Journal of Science and Technology, Transactions of Electrical Engineering, 43(1), $1-12$.

Preishuber, M., Hütter, T., Katzenbeisser, S., and Uhl, A. (2018). Depreciating motivation and empirical security analysis of chaos-based image and video encryption. IEEE Transactions on Information Forensics and Security, 13(9), 2137-2150.

Sambas, A., Gundara, G., Sanjaya, M., and Mamat, M. (2015). Analisis dinamika kompleks sistem Malasoma dan aplikasinya pada speech encryption. ALHAZEN Journal of Physics, 2(2), 1-10.

Sambas, A., Vaidyanathan, S., Zhang, S., Zeng, Y., Mohamed, M. A., and Mamat, M. (2019). A new doublewing chaotic system with coexisting attractors and line equilibrium: bifurcation analysis and electronic circuit simulation. IEEE Access, 7, 115454-115462.

Sambas, A., Vaidyanathan, S., Tlelo-Cuautle, E., Abd-El-Atty, B., Abd El-Latif, A. A., Guillén-Fernández, O, Hidayat, Y., and Gundara, G. (2020). A 3-D multi-stable system with a peanut-shaped equilibrium curve: Circuit design, FPGA realization, and an application to image encryption. IEEE Access, 8, 137116-137132.

Sprott, J. C., and Linz, S. J. (2000). Algebraically simple chaotic flows. International Journal of Chaos Theory and Applications, 5(2), 1-20.

Sukono, Sambas, A., He, S., Liu, H., Vaidyanathan, S., Hidayat, Y., and Saputra, J. (2020). Dynamical analysis and adaptive fuzzy control for the fractional-order financial risk chaotic system. Advances in Difference Equations, 674(1), 1-12.

Vaidyanathan, S., Sambas, A., Mamat, M., and Sanjaya, M. (2017). A new three-dimensional chaotic system with a hidden attractor, circuit design and application in wireless mobile robot. Archives of Control Sciences, 27(4), 541-554.

Varan, M., Yalçın, F., and Uyaroğlu, Y. (2016). Synchronizations and secure communication applications of a third degree Malasoma system with chaotic flow. Optik, 127(23), 11086-11093.

Yin, X., She, J., Liu, Z., Wu, M., and Kaynak, O. (2020). Chaos suppression in speed control for permanentmagnet-synchronous-motor drive system. Journal of the Franklin Institute, 357(18), 13283-13303.

Zhou, S., Li, H., and Zhu, Z. (2008). Chaos control and synchronization in a fractional neuron network system. Chaos, Solitons \& Fractals, 36(4), 973-984. 